\title{
Need We Say More? Measuring Redundancy in Nursing Progress Notes
}

\author{
Danielle Ritz SHALA, BSN, RN, MSc (Health Data Science), MACN ${ }^{\mathrm{a}}$ and \\ Suzanne SHEPPARD-LAW, BN, MPH, PHD ${ }^{\mathrm{a}, \mathrm{b}}$ \\ ${ }^{a}$ Sydney Children's Hospitals Network \\ ${ }^{\mathrm{b}}$ University of Technology Sydney
}

Keywords. Nursing progress notes, redundancy, text similarity, analytics, data science

\section{Introduction}

Progress notes are one of the most common forms of electronic documentation among clinicians, often written as free text narratives of care provided to patients. With the increased adoption of electronic health records to replace paper documentation, studies suggest there has been a growing deterioration in the quality of clinical documentation captured electronically. ${ }^{1,2}$ North American studies on physician clinical documentation have shown that most progress notes are redundant, as information is duplicated from previous documents. ${ }^{2-4}$ This is problematic for quality care as redundant information (1) creates noise that masks new and clinically relevant information, (2) may contain a mixture of outdated information or errors in copy-pasted information, (3) increases the cognitive burden of clinicians, and (4) undermines documentation integrity and patient safety. ${ }^{1}$ While research on electronic health records continues to grow, most studies have focused on physicians' practices. Less is known about the nurse's practices in electronic documentation. Currently, there are no published Australian studies describing text redundancy in the electronic documentation of nursing care. This nurse-led study aimed to measure text similarity (redundancy) in electronic nursing progress notes in an Australian paediatric setting using data science techniques.

\section{Approach}

A retrospective review of nursing progress notes documented in the electronic medical record (eMR) was performed. This study was conducted in the largest Australian paediatric centre in New South Wales, Australia. Records of patients admitted to a Paediatric Intensive Care Unit (PICU) for at least 72 hours between November 2018 and February 2019 were reviewed. Shift summary nursing progress notes, a systematic documentation of patient care containing headings per body system (e.g. CNS/Central Nervous system, CVS/Cardiovascular system, GI/Gastrointestinal system, etc.), were the focus of the study. Progress notes with at least 100 words and authored in PICU by registered nurses were included, while those that are more than 100 words long but refer 
to specific or acute events were excluded. To determine if a note is a shift summary nursing progress note, the progress notes were manually annotated. Text similarity was measured by comparing each shift summary progress note with the previous shift's entry To determine the amount of text that matches between two progress notes, text similarity scores were calculated for pairs of notes and expressed as a percentage using Python $^{5}$, an open-source programming language and data science tool which has increased in popularity in the health sciences due to its large standard library of tools and wide range of functionality including scientific computing and text processing. ${ }^{6}$ Descriptive statistics were used in the analysis.

\section{Body}

A total of 511 shift summary nursing progress notes were compared for text similarity. Overall, the similarity scores of progress notes was widely distributed and positively skewed. Text similarity in the majority $(80 \%, n=409)$ of nursing progress notes was below $20 \%$. Higher similarity scores were also reported, with a total of 32 notes $(6.8 \%)$ having more than $50 \%$ similarity compared with a previously written shift summary nursing progress note. The high frequency of low similarity scores in this study suggests that overall redundancy in the nursing progress notes studied is minimal and there are small sections of the note/text that are often duplicated in nursing narratives. Clarification of electronic documentation guidelines, education on quality nursing documentation, and further research are recommended. Future investigations using natural language processing techniques and qualitative methods are suggested.

\section{Conclusions}

This study demonstrates the leadership of frontline nurses in using analytics to enable a digitally aware workforce. Application of research and data science techniques form a powerful combination in analysing current electronic nursing documentation practice. As this is the first study that we are aware of, this provides insight into the amount of text duplication in electronic nursing notes. This also raises questions around the type of information repeated across multiple notes, and the value it adds to the quality of nursing documentation. Findings can be used to inform future policy and practice.

\section{References}

1. Harrison, M., Koppel, R., \& Bar-Lev, S. (2007). Unintended Consequences of Information Technologies in Health Care--An Interactive Sociotechnical Analysis. Journal Of The American Medical Informatics Association, 14(5), 542-549. doi: 10.1197/jamia.m2384

2. Bowman, S. (2013). Impact of electronic health record systems on information integrity: quality and safety implications. Perspectives in health information management, 10(Fall), 1c.

3. Wrenn, J. O., Stein, D. M., Bakken, S., \& Stetson, P. D. (2010). Quantifying clinical narrative redundancy in an electronic health record. Journal of the American Medical Informatics Association : JAMIA, 17(1), 49-53. 
4. Zhang, R., Pakhomov, S., McInnes, B. T., \& Melton, G. B. (2011). Evaluating measures of redundancy in clinical texts. AMIA Annual Symposium proceedings. AMIA Symposium, 2011, 1612-20. 